\title{
EDITORIAL
}

\section{Utility of intraoperative ultrasound for the resection of focal cortical dysplasia}

\author{
Catherine McClung-Smith, MD \\ Department of Neurological Surgery, Palmetto Health-USC Medical Group, Columbia, South Carolina
}

$\mathrm{E}$ PILEPSY surgery, although still developing today, is probably one of the oldest neurosurgical procedures-evidence of trephination dates back to multiple ancient civilizations. Historically, epilepsy was not considered to be a disease of the brain but rather a demonic possession, and any form of "surgery" or trephination was performed for spiritual or religious reasons rather than medical ones. Even into the 17th and 18th centuries, epilepsy was viewed as a nervous or mental disorder rather than a neurological disease. ${ }^{13}$ The first true surgery for epilepsy was performed in 1886 by Sir Victor Horsley and William Macewen ${ }^{13}$ and surgery was usually for seizures secondary to trauma or tumors. Antiepileptic medications were not readily available at that time, making surgery the only recourse.

With the development of neuroimaging through CT scanning and MRI, many patients with epilepsy were found to have structural lesions. As neuroimaging evolved so did epilepsy treatment, and many patients were found to be candidates for either curative or palliative surgical therapies. The advent of functional imaging including PET scanning and SPECT added to the ability to elucidate the epileptogenic foci. ${ }^{12}$

Currently epilepsy remains a worldwide disease affecting approximately 50 million people. ${ }^{17}$ Epilepsy is categorized as either idiopathic or secondary depending on whether an underlying cause can be found. Focal cortical dysplasia (FCD), a form of secondary epilepsy, is a malformation of cortical development that results in rests of cortical cells that produce an epileptogenic focus. FCD tends to produce early-onset epilepsy that is typically drug resistant and prone to bouts of status epilepticus, and yet it may respond to surgical intervention. ${ }^{4,5,11}$ In fact, in the case of FCD type II, surgery may yield the best results. ${ }^{11}$
The primary predictor of surgical outcome in FCD is the extent of resection. ${ }^{5,11}$ Unfortunately, the offending lesions in FCD are generally difficult to visualize intraoperatively. The authors Noli et al. directly attributed surgical failure to the "difficulties defining the epileptogenic zone in the majority of the patients, and the anatomical location in one." 11 The indistinct boundaries between dysplastic tissue and normal brain as well as the difficulty operating around eloquent cortex probably explains the historically poor outcomes after surgery. Unlike tumor resection, FCD also doesn't lend itself to tactile discrimination because the dysplastic lesion consists of otherwise normal cortical tissue.

The subtypes of FCD were first described in 1971 by Taylor et al. ${ }^{14}$ Since then the subtypes have been modified several times, with the first international consensus in $2011,{ }^{2}$ and a validation paper was published in 2012. ${ }^{10}$ Unfortunately, as the authors Muhlebner et al. concluded, MRI may miss some subtypes of FCD. With the exception of the work done by Muhlebner et al., classifications of FCD are based on histopathological findings rather than imaging characteristics. Adding to the difficulty of resection of FCD is the lack of correlation between degree of dysplasia and regions of epileptogenicity. ${ }^{4}$ The seizures may originate from any point in the dysplastic tissue and, if clear boundaries between the FCD and normal tissue are lacking, the seizure focus may be missed.

Current devices used to identify abnormal cortex in the resection of FCD include visual and tactile information, intraoperative MRI (iMRI), electrocorticography (ECoG), neuronavigation, and intraoperative ultrasound (IOUS). All of these tools have limitations. Visual and tactile information is limited in the resection of FCD, unlike the resection of tumors. iMRI images can be distorted by air, 
metal, inappropriate shielding, or inability to use the device should the patient be unsuitable or should the counts of metallic objects be off. iMRI also comes with a significant cost and will result in delays while the imaging is being obtained. The magnets available range from $0.25 \mathrm{~T}$ to $3 \mathrm{~T}$; however, the imaging obtained is not currently able to undergo postprocessing, thus limiting the utility of the machine. ECoG is invaluable in the surgical treatment of epilepsy; however, this yields 2D information that is functional but not anatomical. Neuronavigation has utility but is currently limited by brain shift. Even as new technology is introduced that may correct for the difficulties with brain shift, neuronavigation will not be able to offer realtime images.

IOUS has been shown to have utility in the resection of both low-grade and high-grade gliomas. ${ }^{3,9,16}$ The imaging obtained with IOUS is real time and some neuronavigational systems offer packages that will allow the ultrasound to link to the navigational system, thus allowing targeting to be performed. ${ }^{9}$ Ultrasound devices may provide $2 \mathrm{D}$ or $3 \mathrm{D}$ imaging depending on the number of views and, although there is a learning curve, this is easily overcome with practice.

Resection goals in oncological surgery parallel epilepsy surgery in the goal of maximal safe resection. The use of IOUS in the resection of low-grade and high-grade gliomas has been shown to be safe and effective. ${ }^{3,9,16} \mathrm{In}$ one case series the use of IOUS was shown to have accuracy close to that of iMRI, ${ }^{3,9}$ and Moiyadi et al. considered IOUS to be a viable alternative to iMRI. Moiyadi et al. also showed that the use of navigable IOUS would allow for safe radical resections of malignant gliomas, and Ulrich et al. showed that a minimal bone flap could still be maintained with use of the IOUS..$^{16}$

Based on the advances in the use of IOUS in oncological neurosurgery, it would stand to reason that the technology could be expanded into other forms of resective surgery. We applaud the authors for expanding upon the works of Miller et al. ${ }^{7.8}$ and Lee et al. ${ }^{6}$ and for providing us with the largest case series to date of FCD resected with the assistance of IOUS. In this prospective case series the authors present 15 consecutive patients who underwent resective surgery for all types of FCD. Since the submission of this manuscript an additional paper describing a case series of 6 patients with FCD type II has been published. These authors also expanded upon the work of Miller et al. and Lee et al., with excellent results; however, the patient population was smaller and limited to FCD type II. ${ }^{15}$

The publication by Tringali et al. paralleled this work by Akeret et al., in that the dysplastic lesions in FCD type II were found to have sharp borders easily seen on IOUS and the investigators concluded that IOUS had a resolution similar to that of iMRI. The authors describe excellent surgical results, with the goal of publishing an alternative to iMRI. ${ }^{1,15}$ In this context both Akeret et al. and Tringali et al. are seeking to show the utility of IOUS over iMRI and the ease with which IOUS can be incorporated into the surgical planning.

The authors Akeret et al. also use the IOUS technique to delineate the subtypes of FCD based on imaging. IOUS was also helpful in differentiating FCD from other patho- logical entities such as gliosis, as they found in 1 patient. In all cases in which FCD was found, regardless of subtype, the authors also found clear borders and circumscribed lesions. In the 1 case in which the borders were blurred the patient was found to have gliosis rather than a dysplastic lesion. ${ }^{1}$

Akeret et al. found that in FCD type I lesions the borders were more difficult to define. FCD types IA and IB were identical on IOUS; however, FCD type IC showed increased hyperechogenicity. The authors found FCD type II to have clear borders, as did Tringali et al., but Akeret et al. also described more pronounced abnormalities on IOUS when compared to the other forms of dysplasia, with more prominent abnormalities in FCD type IIB. ${ }^{1}$

The need for preoperative imaging and good patient selection cannot be overstated, and this case series is not meant to replace multimodality patient workup and interdisciplinary discourse. In all cases of surgically treated epilepsy a preoperative hypothesis is needed to ensure that the patient is undergoing the procedure most appropriate to that individual. Epilepsy surgery is meant to be a tailored approach and not a one-size-fits-all procedure. Akeret et al. are careful to discuss the need for preoperative MRI with postprocessing as part of surgical planning. The authors also used ECoG intraoperatively as a part of the multimodality intervention they provided.

Although limited by the subjective nature of sonographic imaging and a small sample size, this article adds to the literature by showing that not only is IOUS useful in delineating the borders of FCD, it is also potentially able to assist in differentiating the subtype. Although the degree of dysplasia does not necessarily correlate with epileptogenic properties, Akeret et al. did find that there was a correlation between the degree of dysplasia and the underlying subtype. This information would be expected to give the surgeon additional information in order to tailor the surgical approach intraoperatively. Because seizure freedom is directly related to the extent of resection, these real-time images may improve the resection outcomes if used as part of a multimodality approach.

https://thejns.org/doi/abs/10.3171/2018.6.FOCUS18329

\section{References}

1. Akeret K, Bellut D, Huppertz HJ, Ramantani G, König K, Serra C, et al: Ultrasonographic features of focal cortical dysplasia and their relevance for epilepsy surgery. Neurosurg Focus 45(3):E5, 2018

2. Blumcke I, Thom M, Aronica E, Armstrong DD, Vinters HV, Palmini A, et al: The clinicopathologic spectrum of focal cortical dysplasias: a consensus classification proposed by an ad hoc Task Force of the ILAE Diagnostic Methods Commission. Epilepsia 52:158-174, 2011

3. Coburger J, Scheuerle A, Thal DR, Engelke J, Hlavac M, Wirtz CR, et al: Linear array ultrasound in low-grade glioma surgery: histology-based assessment of accuracy in comparison to conventional intraoperative ultrasound and intraoperative MRI. Acta Neurochir (Wien) 157:195-206, 2015

4. Fauser S, Huppertz HJ, Bast T, Strobl K, Pantazis G, Altenmueller DM, et al: Clinical characteristics in focal cortical dysplasia: a retrospective evaluation in a series of 120 patients. Brain 129:1907-1916, 2006

5. Jin B, Wang J, Zhou J, Wang S, Guan Y, Chen S: A longitudinal study of surgical outcome of pharmacoresistant epilepsy 
caused by focal cortical dysplasia. J Neurol 263:2403-2410, 2016

6. Lee CC, Lin CF, Yu HY, Hung SC, Shih YH, Hsu SPC: Applications of intraoperative ultrasound in epilepsy surgery for focal cortical dysplasia. J Med Ultrasound 22:43-46, 2014

7. Miller D, Knake S, Bauer S, Krakow K, Pagenstecher A, Sure U, et al: Intraoperative ultrasound to define focal cortical dysplasia in epilepsy surgery. Epilepsia 49:156-158, 2008

8. Miller D, Knake S, Menzler K, Krakow K, Rosenow F, Sure $\mathrm{U}$ : Intraoperative ultrasound in malformations of cortical development. Ultraschall Med 32 (Suppl 2):E69-E74, 2011

9. Moiyadi AV, Shetty PM, Mahajan A, Udare A, Sridhar E: Usefulness of three-dimensional navigable intraoperative ultrasound in resection of brain tumors with a special emphasis on malignant gliomas. Acta Neurochir (Wien) 155:2217-2225, 2013

10. Muhlebner A, Coras R, Kobow K, Feucht M, Czech T, Stefan $\mathrm{H}$, et al: Neuropathologic measurements in focal cortical dysplasias: validation of the ILAE 2011 classification system and diagnostic implications for MRI. Acta Neuropathol 123:259-272, 2012

11. Noli D, Bartuluchi M, González FS, Kaltenmeier MC, Cersosimo R, Rugilo C, et al: Type II focal cortical dysplasia: electroclinical study and surgical outcome in 31 pediatric patients. Childs Nerv Syst 29:2079-2087, 2013

12. Polkey CE: The evolution of epilepsy surgery. Neurol India 65 Suppl:S45-S51, 2017

13. Schijns OE, Hoogland G, Kubben PL, Koehler PJ: The start and development of epilepsy surgery in Europe: a historical review. Neurosurg Rev 38:447-461, 2015
14. Taylor DC, Falconer MA, Bruton CJ, Corsellis JAN: Focal dysplasia of the cerebral cortex in epilepsy. J Neurol Neurosurg Psychiatry 34:369-387, 1971

15. Tringali G, Bono B, Dones I, Cordella R, Didato G, Villani F, et al: Multimodal approach for radical excision of focal cortical dysplasia by combining advanced magnetic resonance imaging data to intraoperative ultrasound, electrocorticography, and cortical stimulation: a preliminary experience. World Neurosurg 113:e738-e746, 2018

16. Ulrich NH, Burkhardt JK, Serra C, Bernays RL, Bozinov O: Resection of pediatric intracerebral tumors with the aid of intraoperative real-time 3-D ultrasound. Childs Nerv Syst 28:101-109, 2012.

17. World Health Organization: Epilepsy fact sheet. WHO.int. February 8, 2018 (http://www.who.int/news-room/fact-sheets/ detail/epilepsy) [Accessed July 11, 2018]

\section{Disclosures}

The author reports no conflict of interest.

\section{Correspondence}

Catherine McClung-Smith: catherine.mcclungsmith@ palmettohealth.org.

\section{INCLUDE WHEN CITING}

DOI: 10.3171/2018.6.FOCUS18329. 